\title{
Etiology, diagnosis, and surgical management of obstructive salivary gland disease
}

\author{
Neeraja Nina Karwowska^, Michael D. Turner^ \\ Division of Oral and Maxillofacial Surgery, Department of Otolaryngology, The Mount Sinai Hospital, New York, NY, USA \\ Contributions: (I) Conception and design: NN Karwowska; (II) Administrative support: MD Turner; (III) Provision of study materials or patients: MD \\ Turner; (IV) Collection and assembly of data: None; (V) Data analysis and interpretation: None; (VI) Manuscript writing: All authors; (VII) Final \\ approval of manuscript: All authors. \\ Correspondence to: Michael D. Turner. 10 Union Square East, Suite 5B, New York, NY 10003, USA. Email: Michael.turner@mountsinai.org; Neeraja \\ Nina Karwowska. 1 Gustave L. Levy Place, New York, NY 10029, USA. Email: Neeraja.karwowska@mountsinai.org.
}

\begin{abstract}
Obstructive salivary gland disease (OSGD) represents a large subset of salivary gland entities that affect a significant number of patients every year. Many different pathologic processes such as sialoliths, mucous plugs, strictures, and ductal stenosis can present with nearly identical obstructive symptoms, making diagnosis and treatment challenging. Current management of OSGD revolves around resolution of acute infection, targeted imaging, and definitive surgical management of the causal obstruction. Technological advances and improved diagnostic methods over the last two decades have led to a paradigm shift in management of these disorders, with an emphasis on gland-sparing and minimally-invasive therapies. Imaging for obstructive salivary gland disorders has shifted away from traditional sialography and plain film radiography in favor of ultrasound, computed tomography (CT), and magnetic resonance imaging (MRI). Rehydration, gland massage, and antibiotics continue to be the mainstay of medical management. The use of sialendoscopy represents a minimally-invasive method to treat most OSGD without the morbidity associated with sialoadenectomy or other open procedures. The development of multiple adjunct treatment methods such as mechanical lithotripsy, laser lithotripsy, and extracorporeal shock wave lithotripsy have added to the overall effectiveness of minimally-invasive treatments for OSGD. This paper presents a review on the four main etiologies of salivary gland obstruction, appropriate diagnostic workup, and medical and surgical management of these disorders.
\end{abstract}

Keywords: Salivary gland; obstruction; sialendoscopy; sialolith, stricture

Received: 01 November 2020; Accepted: 13 April 2021; Published: 10 June 2021.

doi: $10.21037 /$ fomm-20-73

View this article at: http://dx.doi.org/10.21037/fomm-20-73

Obstructive salivary gland disease (OSGD) is a subset of salivary gland disorders that specifically refers to occlusion of a salivary duct with swelling of the associated gland. Though there are many etiologies that can cause OSGD, the most common causes are sialoliths (salivary stones), mucous plugs, strictures, and duct stenosis. This article aims to review the common etiologies of OSGD and discuss proper diagnosis and surgical management of these conditions.

\section{Overview of the major salivary glands}

The human body contains three pairs of major salivary glands: the parotid, submandibular, and sublingual glands. Collectively, these glands produce $90 \%$ of saliva (1). The largest of the three is the parotid gland, which is found in

\footnotetext{
^ ORCID: Michael D. Turner, 0000-0003-1208-9551; Neeraja Nina Karwowska, 0000-0001-7714-5433.
} 
the preauricular area and contains the facial nerve, which separates the gland into deep and superficial lobes. The parotid gland secretes only serous saliva through the parotid duct, sometimes referred to as Stensen's duct. Serous saliva is thin and watery in consistency and has a high protein content (1). The next major salivary gland is the submandibular gland, an encapsulated gland below the angle of the mandible that secretes a mixed serous and mucous saliva. Mucous saliva, in contrast to serous saliva, is thick and viscous, and contains mucin and mucopolysaccharides (1). The submandibular duct, also called Wharton's duct, drains immediately posterior to the mandibular incisor teeth. The sublingual glands are the smallest of the major salivary glands and are found bilaterally in the floor of the mouth above the mylohyoid muscle. The sublingual gland secretes mucous saliva, which exits the gland and enters the oral cavity via Bartholin's excretory duct or the smaller ducts of Rivinus (1). Lastly, there are 600-1,000 minor salivary glands in the oral cavity dispersed amongst the tongue, lip, buccal mucosa, palate, and floor of mouth. These minor salivary glands secrete mostly mucous saliva (1). Obstructions can occur in the sublingual gland, although it is relatively rare compared the submandibular or parotid glands (2). For the purposes of brevity and clinical relevance, this article will focus on OSGD of the parotid and submandibular glands and their associated ducts.

\section{Etiology of salivary gland obstruction}

\section{Sialoliths}

The most frequent etiologies of OSGD are sialoliths, mucus plugs, strictures, and stenoses (2). Sialolithiasis, or the presence of salivary stones, is the most common etiology of OSGD (3). It preferentially affects the major salivary glands and their associated structures, with $80-90 \%$ of sialoliths located in the submandibular glands, and the remaining $5-20 \%$ occurring in the parotid glands (4). The high predilection for the submandibular gland has been postulated to be a combination of anatomic factors such as the length and course of the submandibular duct as well as chemical factors such as the high mucin and calcium content of the gland (4). Sialolithiasis can affect both left and right sides equally, but rarely is seen bilaterally. If associated with the submandibular gland, sialoliths are found in three distinct regions: the intra-parenchyma, the hilum/proximal duct, and the distal duct (4). The vast majority of submandibular stones (up to $90 \%$ ) are located in the distal third of the duct or at the hilum of the gland rather than an intraparenchymal location (3). In the parotid gland, studies have shown that the majority of stones are in the main duct, with less than $15 \%$ of stones in the hilum (3). Salivary gland stones are composed of organic material mixed with precipitated salts, such as calcium phosphate and calcium carbonate. They can be round, oblong, or irregular and can range from a few millimeters in size up to 2 centimeters or more. The etiologic agents responsible for sialolith formation have yet to be determined, and no clear systemic factors have been definitively linked. Patients with renal stones, for example, have not been found to have a higher risk of developing salivary gland stones (5).

\section{Mucous plugs}

Another etiology of obstruction is the formation of mucous plugs within the salivary gland ducts. For a mucous plug to form, there must be two factors present concurrently: an existing area of salivary stasis, and an increase in the concentration of mucous in the saliva. Salivary stasis, the slowing or stoppage of saliva flow, is a major factor in mucous plug formation. Stasis occurs from either hyposalivation or an anatomic variance resulting in a region of turbulence within the duct system. Polypharmacy is the leading cause of hyposalivation, particularly when the medications include anticholinergics, antihistamines, and antidepressants $(6,7)$.

The submandibular glands have a higher concentration of mucous cells than the parotid glands, and are thus more likely to form obstructive mucous plugs. When a mucous plug is the sole cause of obstruction, it is referred to as a primary mucous obstruction. If a mucous plug occurs in conjunction with another obstructive process, such as a sialolith or a stricture, it is referred to as a secondary mucous obstruction.

\section{Strictures and stenoses}

Strictures and stenoses are also common causes of OSGD. Strictures are defined as a short segment of intraluminal scar or adhesion that create a near-complete blockage of the duct, resulting in an extremely small lumen. In contrast to sialoliths, strictures are more commonly found in the parotid duct (70-75\%), and affect women three times more frequently than men (8). A stenosis is defined as a long segment of circumferential narrowing of the ductal lumen. Stenoses preferentially affect the parotid gland 
and have been subclassified into 3 types. Type 1 stenosis is characterized by inflammatory changes within the stenotic area, whereas type 2 stenosis is associated with circular or weblike changes within the duct. Type 3 stenosis has been described as a purely fibrotic reaction, and represent the vast majority of stenoses (9). The exact etiology of strictures and stenoses is unknown, though prior trauma or surgery in the area have been proposed as predisposing factors. Various associations to allergies, autoimmune disease, radioiodine therapy, and prior head and neck radiation have also been postulated (10).

Any form of obstruction in the gland that decreases or stops normal salivary flow increases the occurrence of a retrograde bacterial contamination from the oral cavity into the gland proper. It is important to note that OSGD can lead to a salivary gland infection, and infections can also lead to an obstruction. These processes of obstruction and infection can exist either independently or concurrently.

\section{Principles of evaluation}

Evaluation of a patient suspected of having an obstructed salivary gland begins with a detailed history. Patients with OSGD generally report a history of recurrent pain and facial swelling in the area of a salivary gland, particularly during mealtime. The pain and swelling are caused by the obstruction of salivary flow and the resultant buildup of saliva proximal to the obstruction. A thorough medical history should be performed to identify systemic conditions that may predispose the patient to OSGD. Cystic fibrosis, HIV, diabetes, and a history of radiation therapy to the head and neck are examples of conditions that are associated with OSGD. Cystic fibrosis is associated with recurrent mucous plugging, while patients with diabetes and HIV have been shown to have severe hyposalivation (11). A thorough medication review should also be conducted to identify polypharmacy, a known cause of hyposalivation. Medication-induced hyposalivation not only leads to increased risk of salivary gland obstruction, but also predisposes patients to increased mucosal fungal infections and an increased caries rate (12).

Other specific components of the history that should be ascertained are associated constitutional symptoms (fever, chills, malaise), onset of symptoms (slow vs. rapid), duration of symptoms, location of symptoms (unilateral vs. bilateral), and history of foul taste and smell. These factors can help determine if a patient has sialadenitis and can aid in creating a differential diagnosis, which may also include viruses and neoplasms.

The physical examination involves careful observation of the face for asymmetry, previous scars, swelling, facial nerve weakness or paralysis, and discrete masses. The major salivary glands should then be inspected for enlargement, erythema, warmth, and discharge. Manual massage of the ducts should be performed and the type of discharge noted (normal saliva, purulence, or lack of saliva). Nearby lymph nodes should be palpated and any lymphadenopathy noted. The overall condition of the patient's dentition should also be evaluated and gross dental decay noted. Physical examination findings, in addition to a thorough history, can direct the practitioner towards the etiology of the obstructive process.

\section{Imaging of OSGD}

Imaging of suspected OSGD has evolved over the last several decades. Plain film and sialography have largely been replaced by ultrasound, magnetic resonance imaging (MRI), computed tomography (CT), and sialendoscopy. There has additionally been a recent trend of performing CT sialography in office as point-of-care cone-beam CT scan technology has become more prevalent. The choice of imaging should be guided by the most likely obstructive cause. For example, suspicion of an acute sialadenitis or a sialolith-related obstruction is best confirmed by ultrasound, the first-line in many parts of the world, or computed tomography, in which the sialolith is visible as a hyperdensity in the gland (13). While CT scan has high sensitivity for detection of sialoliths, the sensitivity for strictures and stenoses is significantly lower. Diagnosis of strictures and stenoses is best made with ultrasound, sialography, or endoscopy. Ultrasound can be utilized for detection of strictures, adhesions, and stones as small as $1.5 \mathrm{~mm}$, though it is technique-sensitive and operatordependent (14). Dilated ducts may be identified with ultrasound and then traced distally to find the narrowing of the lumen. It should be noted that a failure to visualize a dilated duct on ultrasound does not rule out the presence of a stricture or stenosis. In cases where clinical suspicion is high despite the absence of a positive finding on ultrasound, further imaging and diagnostic workup is indicated.

If the underlying obstructive etiology is equivocal, sialendoscopy, a minimally-invasive endoscopic procedure, can be considered. Sialendoscopy, which is both diagnostic and therapeutic, involves the introduction of a small 0.8 to $1.6 \mathrm{~mm}$ semirigid endoscope into the duct after careful 
sequential dilation of the duct (15). The primary advantage with sialendoscopy is the ability to directly visualize the inner lumen of the salivary ducts and the mucosal lining. Sialendoscopy can definitively differentiate between mucous plugs, sialoliths, stenosis, and strictures, as well as other rare entities such as foreign body impactions.

\section{Medical management of OSGD}

Medical management of OSGD revolves around common tenets of treatment regardless of the obstructive etiology. First, patients who have developed acute bacterial sialadenitis as a result of any obstructive cause should be treated with antibiotics prior to surgical intervention in order to reduce inflammation and tissue friability. As most bacterial sialadenitis is due to gram positive (particularly S. aureus) and anaerobic bacteria, antibiotics with broadspectrum coverage such as amoxicillin-clavulanic acid should be prescribed (16). If antibiotics fail to resolve the infection or a discrete abscess forms, incision and drainage is indicated if the purulence does not spontaneously drain from the duct. Once the acute infection has resolved, surgical removal of the sialolith should be performed. The specific method and technique is dependent upon stone location, shape, and size, and will be discussed in detail in the next section.

Massaging of the gland, increased hydration, and sialagogues are also recommended to promote serous saliva production. It should be noted that sialagogues are not useful in patients with complete obstruction of the duct (e.g., from a large sialolith), as the saliva has no drainage pathway; in these cases, increased saliva may only further exacerbate symptoms of pain and swelling.

\section{Surgical management of OSGD}

Surgical management of OSGD has undergone a marked shift over the past two decades. The morbidity associated with sialadenectomy and open surgery led to the progressive development of minimally invasive, endoscopic methods of treatment. The introduction of specialized miniaturized instruments and use of adjunct therapies such as intracorporeal and extracorporeal lithotripsy have further increased the success rate of minimally-invasive management of OSGD. While traditional open surgery is still the mainstay of treatment for conditions such as salivary neoplasms, endoscopic treatment is now the firstline therapy for most cases of sialolithiasis and other ductal obstruction. Combined approaches that involve the use of endoscopy in addition to transoral or transcutaneous incisions are also useful in the treatment of OSGD.

\section{Surgical management of mucous plugs}

While many mucous plugs are cleared spontaneously or after medical management, some are tenacious and require mechanical disruption via hydrodissection, lacrimal probes and dilators, or sialendoscopy. Ultimately, plugs that are not dislodged and remain in the ductal system can calcify and become sialoliths.

\section{Surgical management of sialolithiasis}

Submandibular stones that can be palpated manually in the floor of the mouth (in the distal 1/3 of the duct) can be excised with an incision directly over the duct (transoral duct slitting) after administration of local anesthesia. Duct patency can be compromised in patients with sialolithiasis as the proximal region behind the sialolith is often fibrosed from chronic infection and inflammation. Therefore, sialendoscopy should be performed immediately after direct excisional removal of the stone to ensure patency of the duct and confirm the absence of additional sialoliths or sialolith fragments.

Sialoliths that are more proximal and cannot be removed under direct vision can removed with a solely endoscopic technique (17), with the approach dependent on the size of the sialolith. Stones under $5 \mathrm{~mm}$ in diameter may be removed intact and intraductally with the sialoendoscope and accessory instruments, such as retrieval baskets or miniforceps (Figure 1). The first step in sialendoscopy is atraumatic dilation of the orifice of the duct. Lacrimal probes starting at 4-0 are gently advanced into the duct and the duct dilated with gentle circular motions. The probe size is gradually increased with the dilators until the endoscope can be passed freely (Figure 2). A small papillotomy is sometimes made in cases where the probes cannot be easily introduced through the natural orifice of the duct. After successful dilation, the endoscope is inserted through the duct, taking care not to perforate through the duct walls. Saline is infiltrated through the irrigation port, which further dilates the duct, creates an optical cavity, and concurrently displaces mucous plugs and debris. Small instruments such as baskets and forceps may then be inserted through the working ports of the sialoendoscope to aid in further treatment (18). Once the basket or forceps 


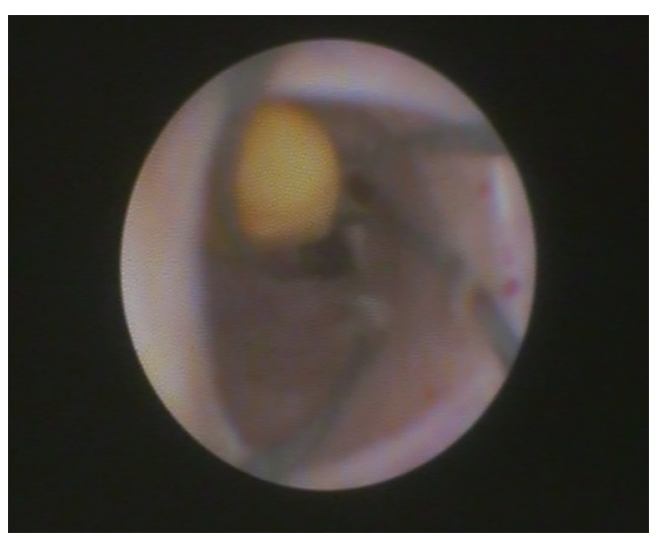

Figure 1 Removal of a submandibular sialolith utilizing a retrieval basket through a sialoendoscope.

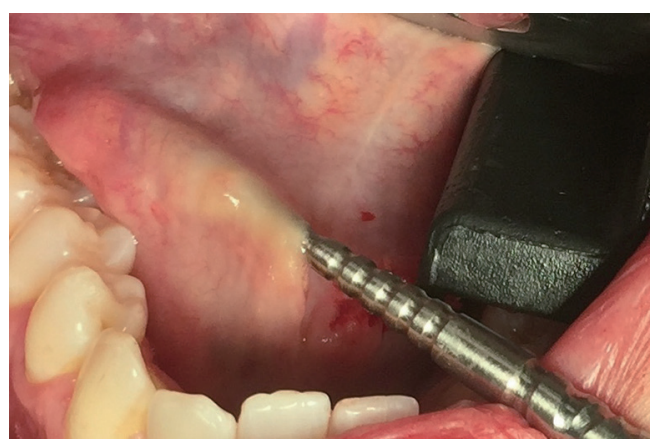

Figure 2 Dilation of right submandibular duct prior to introduction of sialoendoscope.

have engaged the sialolith, the instrument should be gently retracted proximally, with care taken not to tear or avulse the ductal lumen with overaggressive force. If the stone is unable to be endoscopically retrieved in a single piece, the adjunctive modality of lithotripsy may be attempted (19). Lithotripsy, which can be intracorporeal or extracorporeal, involves fragmentation of the sialolith within the duct or gland, followed by irrigation of the stone fragments out of the ductal system.

\section{Intracorporeal lithotripsy}

Intracorporeal lithotripsy, also referred to as endoscopic lithotripsy, is performed in multiple ways, including mechanical fragmentation or with use of lasers or pneumatic devices (19). Mechanical fragmentation of a sialolith can be achieved by use of a grasping forceps through the endoscope, which crushes the stone into smaller fragments. The grasping forceps is then used to remove any large pieces of the sialolith. Any remaining small granules will either become fibrosed into the mucosa of the duct or gland, or be self-expressed in the patient's saliva over the next 1-2 months (19).

Intracorporeal lithotripsy of the sialolith can also be performed utilizing a variety of different lasers, a technique originally developed for treatment of nephrolithiasis. The most common lasers used are holmium and erbium: YAG lasers. With this technique, the laser fiber is deployed through the working channel of the sialoendoscope and placed in contact with the sialolith surface. The theory behind laser lithotripsy is that the absorbed energy from the laser pulses causes the formation of an expanding cavity of ions and electrons on the sialolith surface, leading to high-pressure shock waves that fragment the stones (19). The results from laser lithotripsy are variable for many reasons, one of which is the chemical composition of the sialoliths. Energy absorption is material-dependent, and sialoliths are mostly comprised of calcium phosphate and carbonate in addition to organic material like glycoproteins, mucopolysaccharides, and cellular debris. Due to the composition, only $60 \%$ of the shock waves from the lasers penetrate the stone, while the remainder of energy is reflected and scattered away from the sialolith (19). This phenomenon occurs particularly with the holmium laser and results in thermal damage and necrosis of the surrounding tissue. Inadvertent laser damage can also result in ductal perforation or adhesion formation. Additionally, because the tip of the endoscope is in close proximity to the laser fiber and the sialolith, the heat generated at the surgical site can result in damage to the endoscope itself, rendering it unusable. One other major limitation of laser lithotripsy is that it is very technique sensitive and often time-consuming, especially in cases involving multiple sialoliths. Despite these pitfalls, some studies have reported stone extraction success rates of over $80 \%(19,20,21)$.

Intracorporeal pneumatic lithotripsy is yet another method of fragmenting calculi. Pneumatic lithotripsy is based on the transmission of kinetic energy from the probe to the surface of a sialolith (19). To perform this technique, the lithotripter is advanced to the sialolith and placed in contact with the stone surface. A pellet is then propelled forward within the probe, where it strikes the inner, distal portion of the probe tip. The kinetic energy is then transmitted to the sialolith, fracturing it. Microforceps and baskets are used to remove the smaller fragments. There are multiple limitations to this technique. The first is that the sialolith or its fragments can be driven proximally and 


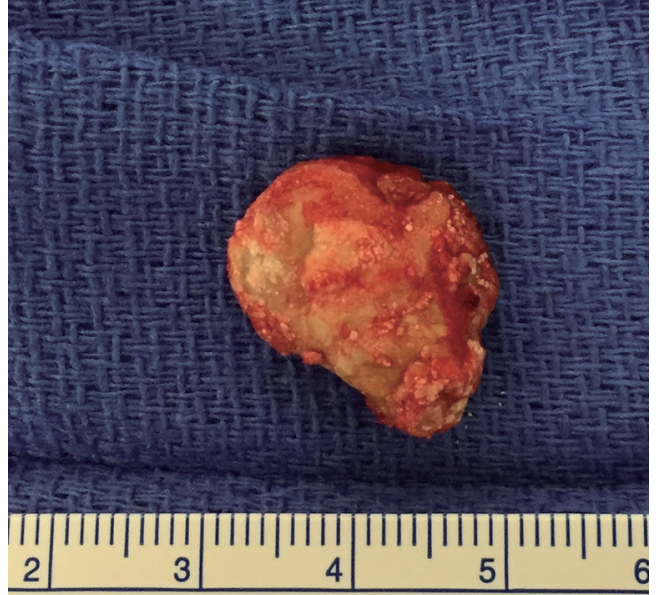

Figure 3 Submandibular megalith.

embed themselves in smaller ducts, which can render them irretrievable. If these small fragments are contaminated with bacteria, they can cause an acute sialadenitis with abscess formation. If this occurs, sialoadenectomy should be considered. Secondly, similarly to laser lithotripsy, there is a risk of damage to the surrounding tissues. Though there is a paucity of data on these devices, Koch et al. treated 49 stones with a pneumatic lithotripter in one study and successfully achieved complete fragmentation in $98 \%$ of cases (22).

Lastly, various other intracorporeal methods such as intracorporeal electrokinetic lithotripsy and intracorporeal electrohydraulic lithotripsy have been reported in the literature. These techniques have not been well-studied and are not utilized in standard practice. Complications associated with these techniques have resulted in acoustic damage and significant tissue damage, respectively (19).

\section{Extracorporeal lithotripsy}

Extracorporeal lithotripsy, also called extracorporeal shockwave lithotripsy (ESWL), is another treatment modality adapted from urologic treatment of nephrolithiasis. ESWL involves the use of high-intensity acoustic pulses called "shock waves" that are directed at the sialolith; the shock waves are generated by either electromagnetic or piezoelectric sources. After initial location of the stone via ultrasound, a gel-filled probe is placed under the neck or in the area of the gland, and the shock wave pulse is transmitted through the soft tissue. The potential energy of the shock wave is transformed into kinetic energy, causing the stone to shatter from the compressive and tensile forces applied (19). This technique is effective in two clinical scenarios. The first is in the treatment of large salivary stones, including those $15 \mathrm{~mm}$ or larger, which are termed megaliths (Figure 3) (23). Megaliths form over many years, if not decades, before they become symptomatic. Megaliths are typically found within the hilum of the submandibular gland and enlarge proximally into the interstitial tissue. Fibrotic encapsulation is present, and both the sialolith and the lingual nerve can be intimately embedded in the capsule. The lingual nerve can therefore inadvertently be injured during attempts at megalith removal. Extracorporeal lithotripsy can be used to fracture the sialolith away from the capsule, allowing for a more efficient and comprehensive removal. One limitation to using extracorporeal lithotripsy on a megalith is the possibility of shattering the megalith into multiple fragments that are larger than $5 \mathrm{~mm}$ each, which precludes simple endoscopic retrieval. This then requires further lithotripsy, at which point it becomes very difficult to ascertain the overall number of fragments. This may result in fragments being inadvertently retained in the gland, where they can serve as a nidus of infection and promote new sialolith formation.

The second clinical scenario where extracorporeal lithotripsy is indicated is in the management of sialoliths that are $7 \mathrm{~mm}$ or less in diameter and are embedded into the duct wall proper. Lithotripsy can be used to disrupt their contact with the lumen, allowing for easier endoscopic retrieval (24). Reviews of several studies on extracorporeal shockwave lithotripsy have concluded that the effectiveness was $26-69 \%$ for electromagnetic sources $(19,25)$, and 29 $81 \%$ for piezoelectric ESWL, with a higher rate of success in the parotid gland (19). The higher success rate in the parotid gland is thought to be due to the less viscous saliva expressed by the parotid gland (25). In addition, saliva is believed to clear through the horizontally-sloped parotid duct more efficiently than the submandibular duct, which is long, convoluted, and has a superiorly directed path with multiple kinks and bends (26).

\section{Combined approaches to sialoliths}

In the submandibular gland, if the sialolith is not successfully extracted with a solely sialoendoscopic technique or the stone is greater than $5 \mathrm{~mm}$ in diameter, a combined sialolithectomy procedure should be considered. In these cases, the sialoendoscope is advanced into position and the light used to identify and illuminate the sialolith's location (Figure 4). For the submandibular gland, a floor of the mouth incision is made through the superficial 


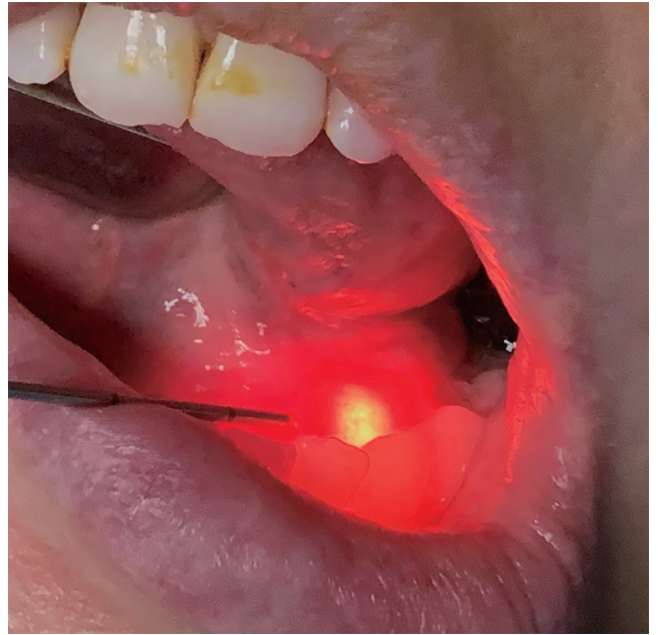

Figure 4 Transillumination of the floor of the mouth with a sialoendoscope prior to performing a transoral sialolithectomy.

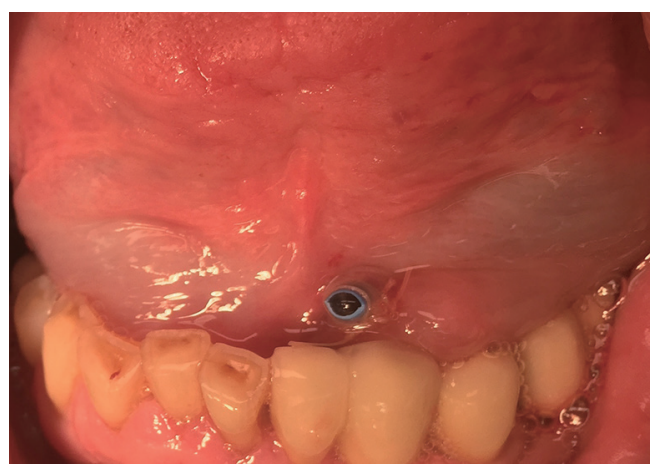

Figure 5 Salivary duct drain to prevent orifice stenosis.

mucosa followed by blunt dissection to the level of the stone. Caution should be taken to locate and retract the lingual nerve to prevent injury to the nerve (27). While any manipulation of the lingual nerve can result in a sensory change, full recovery is expected as long as the endoneurium or the fascicles are not damaged.

For large parotid stones that are not amenable to endoscopic retrieval alone, a transfacial dissection down to the parotid gland capsule is necessary (27). The sialoendoscope is introduced into the duct intraorally and the stone is located. Transillumination is used to locate the stone and a transfacial dissection is made through the capsule and into the gland. The stone is then removed under direct visualization. With this technique, the sialolith is manipulated by pressing against the surface of the stone and pushing it to a more superficial location. This limits the depth needed for the parotid dissection. If the sialolith is located in the deep lobe of the parotid, or even deeply in the superficial lobe, a facial nerve injury can occur. To limit the possibility of a facial paralysis, facial nerve monitoring should be utilized. One of the advantages of an endoscopically-assisted sialolithectomy is that it can easily be converted to a superficial or total parotidectomy if indicated. Upon removal of the gland, the gland should be incised and the stone identified within the tissue, particularly after a superficial parotidectomy. If the sialolith is not retrieved, intraoperative evaluation with plain films or portable CT is used to determine the presence and location of the retained sialolith. If the sialolith is still present, further dissection or conversion to a total parotidectomy should be contemplated.

After successful sialolith removal, regardless of the technique, the duct is injected with steroids, such as 100 milligrams of hydrocortisone or its equivalent (26). A drain should be inserted into the duct for a minimum of 2 weeks, or until the mucosa has healed. The purpose of the drain is to maintain duct patency and to passively dilate the duct to prevent stricture formation. This drain is sometimes referred to as a stent (Figure 5), though the term drain is more appropriate since a "stent" refers to a device that is meant to be permanently retained.

\section{Complications associated with endoscopic sialolith removal}

While complication rates are significantly lower with endoscopy than with open approaches, they do still occur. The most common complication is temporary postoperative gland swelling, which occurs in $5-10 \%$ of cases in the parotid gland and $7-12 \%$ in the submandibular gland (28). Major complications are defined as iatrogenic damage requiring a secondary procedure; these include secondary strictures, duct avulsion, salivary duct perforations and fistulas, lingual nerve injuries, and injury to the lingual gland or ducts resulting in ranula formation $(28,29)$. Secondary strictures of the salivary duct can be diagnosed when there is presence of an ongoing swelling of the salivary gland and an absence or reduction in salivary flow despite sialolith removal (29). The overall risk of post-operative strictures has been reported as less than $3 \%(28,29)$. Dilation of the duct appears to be successful at treating secondary stenosis in most reported cases (29). Duct avulsion is another potential complication of endoscopy, though it has rarely been reported in the literature (28). This complication is primarily associated 
with excessive force being applied during the use of retrieval baskets, and can be mitigated by using gentle manipulation of the basket and endoscope (Figure 6). Surgeon inexperience is a contributing factor to duct avulsion (29). In both parotid and submandibular glands, duct avulsion has a reported occurrence of $0.5 \%$ (29). In the parotid gland, it occurs when a fibrosed salivary gland stone or a large diameter stone $(>5 \mathrm{~mm})$ proximal to the masseteric bend is engaged with a retrieval basket. Currently, there is no salvage surgery reported for this complication and either a superficial or complete parotidectomy is necessary. In the submandibular gland, the salvage procedure is either a complex sialodochoplasty or sialoadenectomy. Perforation and lingual nerve injuries are very rare; perforation has a reported incidence of only $0.5 \%$ in both the parotid and submandibular glands (29), and lingual nerve injury has been reported as between 0.4 and $0.7 \%$ in published studies $(29,30)$. The last of the major complications is ranula formation. Ranulas occur after procedures in the floor of the mouth, including endoscopic treatment of the submandibular gland. One review by Nahlieli et al. reported ranula occurrence in $2.45 \%$ of cases (30); the majority of the cases were treated successfully with marsupialization.

In addition to being minimally morbid, endoscopic surgery has proven to be highly successful for stone removal; one classic study published an $86 \%$ overall success rate for parotid endoscopic sialolithectomy, and an $89 \%$ success rate for submandibular endoscopic sialolithectomy $(14,15)$. Studies have shown that inflamed salivary glands show an improvement in function after the obstructive cause is managed, even in chronic cases (17).

\section{Sialoadenectomy and open approaches}

In cases where minimally-invasive methods fail, or if the stone is located in the gland parenchyma itself and is symptomatic, a sialoadenectomy is indicated. However, removal of the submandibular gland can cause up to a $50 \%$ reduction in saliva production, which is especially problematic in patients with existing hyposalivation. Sialoadenectomy in this population predisposes patients to rampant dental decay, candidiasis, and oral mucosal ulceration (6). Removal of the submandibular gland also poses a risk to the marginal mandibular nerve, which is often encountered during the dissection to the gland and can cause a permanent facial weakness. Injuries to the hypoglossal nerve and lingual nerve have also been reported (27). Sialoliths in the parotid gland can be treated with superficial or total parotidectomy, the latter of which is preferred to

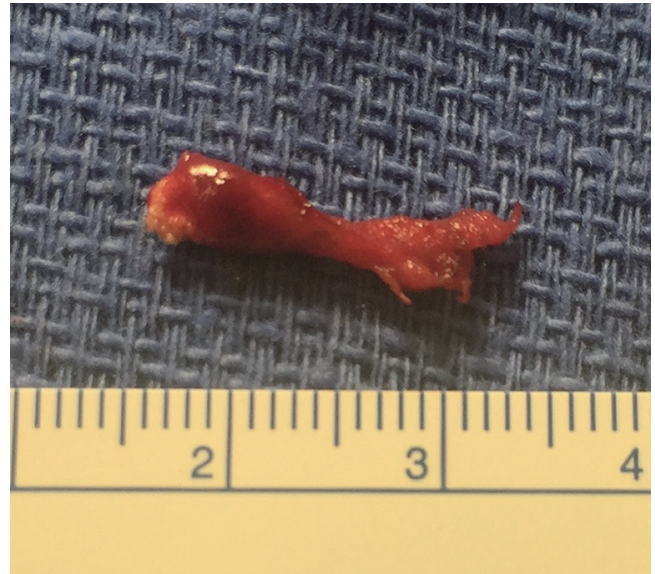

Figure 6 Avulsed submandibular duct while performing a sialolithectomy.

avoid further recurrence.

Facial nerve injury is the main complication associated with superficial parotidectomies, with the literature reporting temporary weakness in $16-38 \%$ of cases and permanent damage in $9 \%$ of cases (27). Facial scarring, sialocele formation, and Frey's syndrome are also potential complications (29). Multiple studies have demonstrated the superiority of sialendoscopy over sialoadenectomy in stone clearance, gland preservation, complication rate, symptom resolution, and recovery time $(24,29)$.

\section{Surgical management of stenosis and strictures}

Stenoses and strictures are also implicated in OSGD and managed in a similar fashion. Important considerations for therapy are location, number of stenoses, length of stenoses, and character of tissue in the stenotic area (31). Sialendoscopy is superior to other forms of imaging as it can directly visualize the ductal walls and differentiate inflammatory stenosis from fibrous stenosis.

Cases of inflammatory ductal stenosis are treated with endoscopic irrigation and intraductal steroid administration $(26,31)$. Fibrous stenoses additionally require sequential dilatation and/or balloon dilatation (32). When sequentially dilating the duct, care should be taken not to perforate the duct wall, which occurs more frequently when utilizing smaller dilators. One clinical sign of perforation is sudden bleeding through the orifice of the duct. In the submandibular gland, another sign of perforation is swelling in the floor of the mouth; this occurs when hydrodissection is attempted and the saline extravasates out of perforations 
in the duct. Aggressive irrigation can lead to severe tissue edema and even obstruction of the airway. A similar process in the parotid gland can occur during insufflation of the region where the duct passes between the masseter and the buccinator muscles. If duct perforation and fluid extravasation occur, the procedure should be discontinued in most cases. If the operator is technically proficient with sialendoscopy, an attempt can be made to navigate from the distal duct past the perforation, and into the proximal duct. A drain should then be placed over the sialoendoscope or guide wire.

Assuming dilation of the duct is successful and the duct is intact, a balloon can be introduced into the duct. The balloon is then inflated along the length of the stenosis. Similarly, if treating a stricture, the balloon is maneuvered within the narrow region and insufflated. To avoid overdilation and duct rupture, it is essential to have either direct or ultrasound-guided visualization of the region prior to and during dilation.

Regardless of the type of narrowing (stricture $v s$. stenosis), drain placement at the conclusion of the procedure is critical in maintaining the patency of the duct during initial healing. Results of endoscopic treatment and dilatation have shown excellent success, with $85-95 \%$ of patients requiring no further therapy (26). In cases of stricture or stenosis where endoscopic treatment fails, open treatment such as transoral duct slitting may be considered (32).

Obstructive pathology such as sialoliths, mucous plugs, strictures and stenoses constitute a large subset of OSGD. Patients with OSGD often present with specific history and/or symptoms that aid in differentiation between the various etiologies. Modern management of OSGD revolves around rehydration, resolution of acute infection, targeted imaging, and definitive surgical management of the causal obstruction. Surgical treatment of OSGD has undergone a paradigm shift in the last two decades, with a current emphasis on minimally-invasive and gland-sparing techniques. Sialendoscopy is a minimally-invasive diagnostic and therapeutic modality that avoids the morbidity associated with sialoadenectomy and can successfully treat many causes of salivary gland obstruction.

\section{Acknowledgments}

Funding: None.

\section{Footnote}

Conflicts of Interest: Both authors have completed the
ICMJE uniform disclosure form (available at https://fomm. amegroups.com/article/view/10.21037/fomm-20-73/coif). The authors have no conflicts of interest to declare.

Ethical Statement: The authors are accountable for all aspects of the work in ensuring that questions related to the accuracy or integrity of any part of the work are appropriately investigated and resolved.

Open Access Statement: This is an Open Access article distributed in accordance with the Creative Commons Attribution-NonCommercial-NoDerivs 4.0 International License (CC BY-NC-ND 4.0), which permits the noncommercial replication and distribution of the article with the strict proviso that no changes or edits are made and the original work is properly cited (including links to both the formal publication through the relevant DOI and the license). See: https://creativecommons.org/licenses/by-nc-nd/4.0/.

\section{References}

1. de Paula F, Teshima THN, Hsieh R, et al. Overview of Human Salivary Glands: Highlights of Morphology and Developing Processes. Anat Rec (Hoboken) 2017;300:1180-8.

2. Carlson ER. Diagnosis and management of salivary gland infections. Oral Maxillofac Surg Clin North Am 2009;21:293-312.

3. Sigismund PE, Zenk J, Koch M, et al. Nearly 3,000 salivary stones: some clinical and epidemiologic aspects. Laryngoscope 2015;125:1879-82.

4. Lustmann J, Regev E, Melamed Y. Sialolithiasis. A survey on 245 patients and a review of the literature. Int J Oral Maxillofac Surg 1990;19:135-8.

5. Choi HG, Bang W, Park B, et al. Lack of evidence that nephrolithiasis increases the risk of sialolithiasis: A longitudinal follow-up study using a national sample cohort. PLoS One 2018;13:e0196659

6. Turner MD, Ship JA. Dry mouth and its effects on the oral health of elderly people. J Am Dent Assoc 2007;138 Suppl:15S-20S.

7. Ship JA, Pillemer SR, Baum BJ. Xerostomia and the geriatric patient. J Am Geriatr Soc 2002;50:535-43.

8. Ngu RK, Brown JE, Whaites EJ, et al. Salivary duct strictures: nature and incidence in benign salivary obstruction. Dentomaxillofac Radiol 2007;36:63-7.

9. Koch M, Iro H. Extended and treatment-oriented classification of parotid duct stenosis. Laryngoscope 
2017;127:366-71.

10. Jackson EM, Walvekar RR. Surgical Techniques for the Management of Parotid Salivary Duct Strictures. Atlas Oral Maxillofac Surg Clin North Am 2018;26:93-8.

11. Shanti RM, Aziz SR. HIV-associated salivary gland disease. Oral Maxillofac Surg Clin North Am 2009;21:339-43.

12. Turner M, Jahangiri L, Ship JA. Hyposalivation, xerostomia and the complete denture: a systematic review. J Am Dent Assoc 2008;139:146-50.

13. Ugga L, Ravanelli M, Pallottino AA, et al. Diagnostic work-up in obstructive and inflammatory salivary gland disorders. Acta Otorhinolaryngol Ital 2017;37:83-93.

14. Capaccio P, Torretta S, Ottavian F, Sambataro G, Pignataro L. Modern management of obstructive salivary diseases. Acta Otorhinolaryngol Ital 2007;27:161-72.

15. Nahlieli O, Nakar LH, Nazarian Y, et al. Sialoendoscopy: A new approach to salivary gland obstructive pathology. J Am Dent Assoc 2006;137:1394-400.

16. Brook I. The bacteriology of salivary gland infections. Oral Maxillofac Surg Clin North Am 2009;21:269-74.

17. Nahlieli O, Neder A, Baruchin AM. Salivary gland endoscopy: a new technique for diagnosis and treatment of sialolithiasis. J Oral Maxillofac Surg 1994;52:1240-2.

18. McCain JP, Montero J. Surgical Retrieval of Submandibular Stones. Atlas Oral Maxillofac Surg Clin North Am 2018;26:111-7.

19. Capaccio P, Torretta S, Pignataro L, et al. Salivary lithotripsy in the era of sialendoscopy. Acta Otorhinolaryngol Ital 2017;37:113-21.

20. Phillips J, Withrow K. Outcomes of Holmium LaserAssisted Lithotripsy with Sialendoscopy in Treatment of Sialolithiasis. Otolaryngol Head Neck Surg 2014;150:962-7.

21. Sionis S, Caria RA, Trucas M, et al. Sialoendoscopy with and without holmium:YAG laser-assisted lithotripsy in the management of obstructive sialadenitis of major salivary glands. Br J Oral Maxillofac Surg 2014;52:58-62

doi: $10.21037 /$ fomm-20-73

Cite this article as: Karwowska NN, Turner MD. Etiology, diagnosis, and surgical management of obstructive salivary gland disease. Front Oral Maxillofac Med 2021;3:17.
22. Koch M, Mantsopoulos K, Schapher M, et al. Intraductal pneumatic lithotripsy for salivary stones with the StoneBreaker: Preliminary experience. Laryngoscope 2016;126:1545-50.

23. Walvekar RR, Tyler PD, Tammareddi N, et al. Roboticassisted transoral removal of a submandibular megalith. Laryngoscope 2011;121:534-7

24. Zenk J, Koch M, Klintworth N, et al. Sialendoscopy in the diagnosis and treatment of sialolithiasis: a study on more than 1000 patients. Otolaryngol Head Neck Surg 2012;147:858-63.

25. Desmots F, Chossegros C, Salles F, et al. Lithotripsy for salivary stones with prospective US assessment on our first 25 consecutive patients. J Craniomaxillofac Surg 2014;42:577-82.

26. Nahlieli O, Shacham R, Yoffe B, et al. Diagnosis and treatment of strictures and kinks in salivary gland ducts. J Oral Maxillofac Surg 2001;59:484-90; discussion, 490-2.

27. Turner MD. Combined Surgical Approaches for the Removal of Submandibular Gland Sialoliths. Atlas Oral Maxillofac Surg Clin North Am 2018;26:145-51.

28. Nahlieli O. Complications of sialendoscopy: personal experience, literature analysis, and suggestions. J Oral Maxillofac Surg 2015;73:75-80.

29. Nahlieli O. Complications of traditional and modern therapeutic salivary approaches. Acta Otorhinolaryngol Ital 2017;37:142-7.

30. Nahlieli O. Advanced sialoendoscopy techniques, rare findings, and complications. Otolaryngol Clin North Am 2009;42:1053-72, Table of Contents.

31. Koch M, Iro H. Salivary duct stenosis: diagnosis and treatment. Acta Otorhinolaryngol Ital 2017;37:132-41.

32. Koch M, Zenk J, Iro H. Algorithms for treatment of salivary gland obstructions. Otolaryngol Clin North Am 2009;42:1173-92, Table of Contents. 